\title{
Mupirocin ointment with and without chlorhexidine baths in the eradication of Staphylococcus aureus nasal carriage in nursing home residents
}

\author{
Chatrchai Watanakunakorn, MD, FACP, FCCP \\ Claudia Axelson, RN, BSN \\ Brenda Bota, BS, MBA, MT(ASCP) \\ Charlotte Stahl, RN, MSN, CIC \\ Youngstown and Rootstown, Ohio
}

Background: Mupirocin ointment has been shown to be effective in eradicating Staphylococcus aureus nasal carriage in residents of a long-term care facility. Antiseptic soaps have been used as adjunct to this therapy. We compared the efficacy of short-term intranasal mupirocin ointment with and without chlorhexidine baths in the eradication of $S$. aureus nasal carriage with follow-up for 12 weeks.

Methods: Residents in four nursing homes known to have endemic methicillin-resistant $S$. aureus were screenied for nasal carriage of $S$. aureus. Residents who had anterior nares cultures positive for $S$. aureus on two separate occasions were divided into two groups. Both groups received intranasal mupirocin ointment twice daily for 5 days and one group also received chlorhexidine baths for the first 3 days. Cultures of anterior nares, axilla, and groins were performed before treatment and 1 day and 1, 4, 8, and 12 weeks after treatment.

Results: After treatment, S. aureus nasal carriage was eradicated in all residents.

Recolonization with $S$, aureus had occurred at 12 weeks in $24 \%$ of residents receiving mupirocin ointment alone $(6 / 25)$ and in $15 \%$ of residents receiving mupirocin ointment plus chlorhexidine baths (4/27).

Conclusions: A short course of mupirocin ointment was effective in eradicating nasal carriage of $S$. aureus in nursing home residents. There were no statistical differences in efficacy between the two regimens with respect to the eradication of nasal carriage and prevention of recolonization with $S$. aureus. (AJIC AM J INFECT CONTROL 1995;23:

$306-9$ )

From the Infectious Disease Section and the Infection Contro Services, St. Elizabeth Hospital Medical Center, Youngstown, and the Northeastern Ohio Universities College of Medicine, Rootstown.

Supported in part by the Department of Internal Medicine Research and Education Fund, Inc., St. Elizabeth Hospital Medical Center Youngstown, Ohio.

Reprint requests: C. Watanakunakorn, MD, St. Elizabeth Hospital Medical Center, P.O. Box 1790, Youngstown, OH 44501-1790.

Copyright (c) 1995 by the Association for Professionals in Infection Control and Epidemiology, Inc.

0196-6553/95 $\$ 5.00+0 \quad \mathbf{1 7 / 4 7 / 6 4 4 0 2}$
Some residents in nursing homes are nasal carriers of Staphylococcus aureus, which may be methicillin susceptible (MSSA) or methicillin resistant (MRSA). MRSA nasal carriers in a longterm care facility have been found to have staphylococcal infections more frequently than noncarriers. ${ }^{1}$ Moreover, MRSA nasal carriers in nursing homes are reservoirs for MRSA when they are transferred to acute care hospitals., ${ }^{2,3}$

Through the years, eradication of $S$. aureus nasal carriage has been attempted with systemic 
and topical antimicrobial agents, with varying degrees of success..$^{4-6}$ Mupirocin ointment has been used with success to treat nursing home residents for nasal carriage, but recolonization occurs. ${ }^{7-8}$ To eradicate $S$. aureus carriage on other parts of the body, which may be the source of recolonization, we applied intranasal mupirocin ointment and gave chlorhexidine baths to patients undergoing long-term hemodialysis who were $S$. aureus nasal carriers, with good success. ${ }^{9}$ The purpose of this investigation was to compare the efficacy of intranasal mupirocin ointment alone with that of the combination of intranasal mupirocin ointment and chlorhexidine baths in the eradication of $S$. aureus nasal carriage in residents of nursing homes.

\section{METHODS \\ Patient population}

Nursing homes in the greater Youngstown area with MRSA colonization known to be endemic among their residents were contacted. The rationale for doing the study was explained to the administrators or directors of nursing to seek their approval for participation in this study. The nursing homes incurred no cost for the study. We provided the medications and performed all the cultures. One of us (C. A.) gave in-service programs and provided written instructions to the nursing home staff regarding proper application of mupirocin ointment to the anterior nares and proper bathing of the whole body with chlorhexidine from the neck down, with special attention to the axillary and groin areas. Permission was obtained from attending physicians to talk to residents under their care regarding this study. Written informed consent was obtained from all residents who volunteered for the study. The research protocol was approved by the medical research committee and the institutional review board of St. Elizabeth Hospital Medical Center.

\section{Screening of residents}

Written informed consent was obtained from all residents who were older than 18 years, were not pregnant, and had no history of allergy to mupirocin or chlorhexidine. Swabs from both anterior nares were obtained for culture. On day 3 , residents whose anterior nares cultures had grown $S$. aureus underwent culture again from both anterior nares and also from axillary and
Table 1. Patient characteristics

\begin{tabular}{|c|c|c|}
\hline $\begin{array}{c}\text { Patient } \\
\text { characteristics }\end{array}$ & $\begin{array}{l}\text { Mupirocin } \\
\text { alone }\end{array}$ & $\begin{array}{c}\text { Mupirocin } \\
\text { plus } \\
\text { chlorhexidine }\end{array}$ \\
\hline \multicolumn{3}{|l|}{$\operatorname{Sex}(n o)}$. \\
\hline Male & 8 & 7 \\
\hline Female & 19 & 22 \\
\hline \multicolumn{3}{|l|}{ Age (yr) } \\
\hline Range & $44-49$ & $42-98$ \\
\hline Median & 79 & 78 \\
\hline \multicolumn{3}{|l|}{ Diabetes mellitus (no.) } \\
\hline Insulin-dependent & 3 & 2 \\
\hline Non-insulin-dependent & 2 & 2 \\
\hline \multicolumn{3}{|l|}{$\begin{array}{l}\text { Positive S. aureus culture } \\
\text { (no.) }\end{array}$} \\
\hline Gastric tube site & 2 & 2 \\
\hline Open wound & 1 & 1 \\
\hline \multicolumn{3}{|l|}{ Functional status (no.) } \\
\hline Independent & 2 & 3 \\
\hline Some assistance & 8 & 10 \\
\hline Most assistance & 15 & 13 \\
\hline Confined to bed & 2 & 3 \\
\hline \multicolumn{3}{|l|}{ Room arrangement (no.) } \\
\hline Semiprivate & 23 & 22 \\
\hline Ward & 4 & 7 \\
\hline \multicolumn{3}{|l|}{ S. aureus carriage } \\
\hline MRSA & 16 & 17 \\
\hline MSSA & 11 & 12 \\
\hline
\end{tabular}

groin regions. A third culture of the anterior nares was done on day 5 if the second nares culture failed to grow $S$. aureus. Residents with two nares cultures growing $S$. aureus were enrolled in the study.

\section{Study protocol}

Enrolled residents were examined for open wounds and decubitus ulcers; these were cultured if present. Demographic data, including the residents' functional status, were recorded. Residents were divided into two groups. Alternate residents were enrolled in each group. Residents in the first group were given mupirocin $2 \%$ ointment in polyethylene glycol base, applied to the anterior nares and any open wound that grew $S$. aureus in culture twice daily for 5 days. Residents in the second group were given the same mupirocin treatment with the addition of a bath with chlorhexidine daily for the first 3 days. Appropriate numbers of mupirocin ointment packages and sterile cotton-tipped applicators (and, for patients in the second group a bottle of chlorhexidine solution) were put in a plastic bag labeled with the resident's name and location. This plastic bag was 
Table 2. Results of positive cultures for $S$. aureus

\begin{tabular}{|c|c|c|c|c|c|c|}
\hline \multirow[b]{2}{*}{ Time of culture } & \multicolumn{3}{|c|}{ Mupirocin alone* } & \multicolumn{3}{|c|}{ Mupirocin plus chlorhexidinet } \\
\hline & Anterior nares & Axilla & Groins & Anterior nares & Axilla & Groins \\
\hline Before treatment & $27 / 27$ & $0 / 27$ & $3 / 27$ & $29 / 29$. & $0 / 29$ & $2 / 29$ \\
\hline 1 day after & $2 / 27$ & $0 / 27$ & $0 / 27$ & $0 / 29$ & $0 / 29$ & $1 / 29$ \\
\hline 1 week after & $0 / 27$ & $0 / 27$ & $0 / 27$ & $1 / 29$ & $0 / 29$ & $0 / 29$ \\
\hline 4 weeks after & $2 / 26$ & $0 / 26$ & $1 / 26$ & $2 / 29$ & $0 / 29$ & $2 / 29$ \\
\hline 8 weeks after & $3 / 25$ & $1 / 25$ & $0 / 25$ & $3 / 28$ & $0 / 28$ & $2 / 28$ \\
\hline 12 weeks after & $6 / 25$ & $0 / 25$ & $0 / 25$ & $4 / 27$ & $0 / 27$ & $2 / 27$ \\
\hline
\end{tabular}

*Two residents were discharged during follow-up.

tTwo residents died during follow-up.

given to the responsible personnel to use for that resident. Posttreatment cultures of the anterior nares; axilla, groin and any open wounds were taken at 1 day, 1 week, 4 weeks, 8 weeks, and 12 weeks after completion of treatment.

\section{Microbiology}

Swabs were streaked on mannitol salt agar and incubated at $37^{\circ} \mathrm{C}$ for 48 hours. Mannitolpositive colonies were tested for coagulase. Coagulase-positive colonies were designated as $S$. aureus. All $S$. aureus isolates were tested for susceptibility to methicillin by the standard diskdiffusion test. ${ }^{10}$

\section{RESULTS}

Four nursing homes participated in this study. The $S$. aureus nasal carriage rates among residents were $10.7 \%, 23.4 \%, 26.5 \%$, and $34.1 \%$ in these four nursing homes. The average was $28.2 \%$ (59/209 residents).

Twenty-nine residents were assigned to the first group and 30 were assigned to the second group. One resident in the first group died before treatment and one was discharged during treatment. One resident in the second group was discharged before treatment. Data from 27 residents and 29 residents, respectively, were therefore available for analysis. There were no side effects from mupirocin ointment or chlorhexidine baths.

Table 1 shows characteristics of both groups, including sex distribution, age, number with diabetes mellitus, number with wound cultures positive for $S$. aureus, functional status, room assignment, and susceptibility of $S$. aureus isolates. These were comparable between groups. Table 2 shows the results of cultures for $S$. aureus. Few residents were colonized at the axilla and extremely few residents were colonized in the groins. Both regimens were effective in eradicating nasal carriage of $S$. aureus, but recolonization occurred in both groups. At 12 weeks after treatment, $24 \%(6 / 25)$ versus $15 \%(4 / 27)$ in the mupirocin and mupirocin plus chlorhexidine groups, respectively, were recolonized. In the first group, three isolates were MRSA and three were MSSA; in the second group, three were MRSA and one was MSSA.

\section{DIScussion}

Antimicrobial soaps containing various antiseptics have been used in conjunction with topical or systemic antimicrobial regimens in attempts to eradicate $S$. aureus nasal carriage, without clearly demonstrated efficacy. ${ }^{6}$ In our study of comparable groups of residents, the $S$. aureus nasal recolonization rate at 12 weeks was $24 \%$ in the mupirocin alone group versus $15 \%$ in the mupirocin plus chlorhexidine group. This finding was not statistically different for our small-sample size. We had hoped to study a much larger population; however, we were unable to recruit more nursing homes to participate in our study. To show statistical significance with a difference of this proportion, we would have needed to enroll almost 400 residents in each group.

The efficacy of short-term application of mupirocin ointment in the eradication of $S$. aureus nasal carriage was again demonstrated in our study. The $S$. aureus nasal recolonization rate at 12 weeks was quite low in both mupirocin-treated groups. Although the long-term use of mupirocin ointment has been associated with the development of mupirocin-resistant strains of MRSA, ${ }^{8}$ short-term use is much less likely to do so. A complete infection control program incorporating 
many components is clearly needed for the control of MRSA in long-term care facilities as well as acute care hospitals. ${ }^{11,12}$

We thank SmithKline Beecham, King of Prussia, Pennsylvania, for providing the mupirocin $2 \%$ ointment and Stuart Pharmaceuticals, Wilmington, Delaware, for providing the chlorhexidine solution.

\section{References}

1. Muder RR, Brennen C, Wagener MM, et al. Methicillinresistant staphylococcal colonization and infection in a long-term care facility. Ann Intern Med 1991;114:107-12.

2. Hsu CCS, Macaluso CP, Special L, Hubble RH. High rate of methicillin resistance of Staphylococcus aureus isolated from hospitalized nursing home patients. Arch Intern Med 1988;148:569-70.

3. Strausbaugh LJ, Jacobson C, Yost T. Methicillin-resistant Staphylococcus aureus in a nursing home and affiliated hospital: a four-year perspective. Infect Control Hosp Epidemiol 1993;14:331-6.

4. Darouiche R, Wright C, Hamill R, Koza M, Lewis D, Markowski J. Eradication of colonization by methicillinresistant Staphylococcus aureus by using oral minocycline-rifampin and topical mupirocin. Antimicrob Agents Chemother 1991;35:1612-5.

5. Strausbaugh LJ, Jacobson C, Sewell DL, Potter S, Ward TT. Antimicrobial therapy for methicillin-resistant Staphylococcus aureus colonization in residents and staff of a Veterans Affairs nursing home care unit. Infect Control Hosp Epidemiol 1992;13:151-9.
6. Boyce JM. Methicillin-resistant Staphylococcus aureus in hospital and long-term care facilities: microbiology, epidemiology, and preventive measures. Infect Control Hosp Epidemiol 1992;13:725-37.

7. Cederna JE, Terpenning MS, Ensberg M, Bradley SF, Kauffman CA. Staphylococcus aureus colonization in a nursing home: nasal eradication with mupirocin. Infect Control Hosp Epidemiol 1990;11:13-6.

8. Kauffman CA, Terpenning MS, He X, et al. Attempts to eradicate methicillin-resistant Staphylococcus aureus from a long-term care facility with the use of mupirocin ointment. Am J Med 1993;94:371-8.

9. Watanakunakorn C, Brandt J, Durkin P, Santore S, Bota B, Stahl C. The efficacy of mupirocin ointment and chlorhexidine body scrubs in the eradication of nasal carriage of Staphylococcus aureus among patients undergoing long-term hemodialysis. AJIC Am J Infect Control 1992;20:138-41.

10. National Committee for Clinical Laboratory Standards. Performance standard for antimicrobial disk susceptibility tests-fourth edition: approved standard. NCCLS document M2-A4, vol 10, no 7. Villanova, Pennsylvania: NCCLS, 1990:1-28.

11. Boyce JM, Jackson MM, Pugliese G, et al. Methicillinresistant Staphylococcus aureus (MRSA): a briefing for acute care hospitals and nursing facilities. Infect Control Hosp Epidemiol 1994;15:105-15.

12. Mulligan ME, Murray-Leisure KA, Ribner B, et al. Methicillin-resistant Staphylococcus aureus: a consensus review of the microbiology, pathogenesis, and epidemiology with implications for prevention and management. Am J Med 1993;94:313-28.

\section{Availability of JournaL back issues}

As a service to our subscribers, copies of back issues of AJIC: AMERICAN JournaL OF INFECTION Control for the preceding 5 years are maintained and are available for purchase from the publisher, Mosby, at a cost of $\$ 10.00$ per issue. The following quantity discounts are available: $25 \%$ off on quantities of 12 to 23 , and one third off on quantities of 24 or more. Please write to Mosby-Y.Year Book, Inc., Subscription Services, 11830 Westline Industrial Dr., St. Louis, MO 63146-3318, or call (800) 453-4351 or (314) 453-435 I for information on availability of particular issues. If unavailable from the publisher, photocopies of complete issues are available from University Microforms International, 300 N. Zeeb Rd., Ann Arbor, MI 48106 (313) 761-4700. 